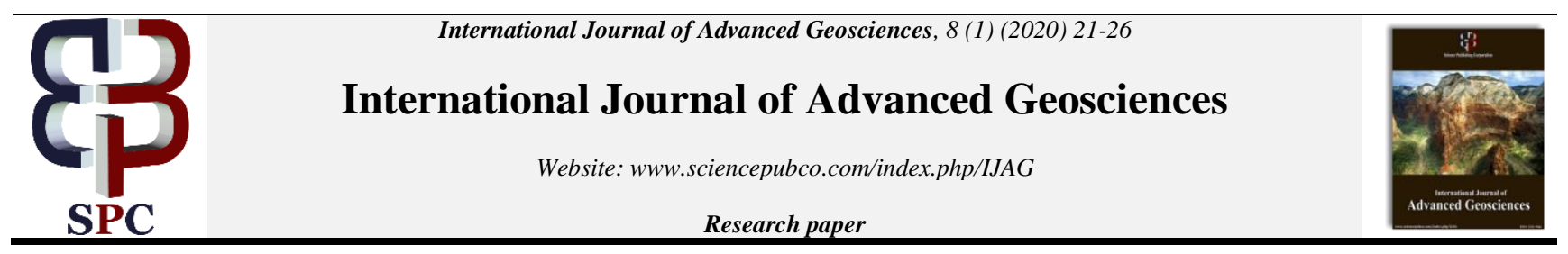

\title{
Morphological and reproductive characterization of developed mutants in groundnut
}

\author{
M. M. A. Mondal ${ }^{1}$, M. S. H. Bhuiyan ${ }^{2 *}$ \\ ${ }^{1}$ Chief Scientific Officer, Bangladesh Institute of Nuclear Agriculture, Mymensingh-2202, Bangladesh \\ ${ }^{2}$ Senior Scientific Officer, Plant Breeding Division, Bangladesh Institute of Nuclear Agriculture, Mymensingh-2202, Bangladesh \\ *Corresponding author E-mail: saikat.ag88@gmail.com
}

\begin{abstract}
Twenty-eight established groundnut mutants and two check cultivars were studied during Kharif-I (March-June) season of 2017 and 2018 to find out their variability and distinct character(s) as identifying keys. All the mutant lines showed erect type sequential branching habits with two seeds in each pod although they had shown significant variability in all vegetative and reproductive structures. According to cluster analysis, 30 mutants/varieties clustered into three major groups at distance level 60 based on the morphological variability of 14 characters. The variability of 14 morphological characters in three principal components was explained by $98.12 \%$ of the total variation. The characters, 100-pod weight had the highest contribution followed by branch length, plant height and 100-kernel weight. Twenty-three mutant genotypes grouped into intermediate type of the extremes in any given identifying key characteristics. Only single genotype of the whole lot showed distinctively the longest primary branch and highest secondary branch number and small seed size (D1/24-29), highest primary branch number (M6/7-25), lowest primary branch number (Mut-2), highest leaflet length and light green leaf colour (Dhaka-1), presence of stem pigmentation and pod beak and highest number of seeds pod-1 (Zhingabadam), leaflet shape lanceolate (M6/54-20). In contrast, only two mutants of the lot showed two buds raceme-1 (M6/36-24 and M6/61-6), bolder pod and seed size and highly constricted pod (Mut-2 and Mut-3). The genotypes with the above distinguished characteristic featured for being ideal genetic markers and could be used in future breeding applications as well as aids in varietal identification.
\end{abstract}

Keywords: Groundnut; Morphology; Variability; Multivariate Analysis; Key Identification.

\section{Introduction}

Groundnut (Arachis hypogaea L.) is an annual legume grown primarily for high quality edible oil (36-54\% on dry matter basis) and easily digestible protein $(12-36 \%)$ in its seeds. It is cultivated worldwide in tropical, sub-tropical and warm temperature areas located between $40^{\circ} \mathrm{N}$ to $40^{\circ} \mathrm{S}$ with world production of 36.9 million tonnes from an area of 25.2 million ha (FAO, 2016). Groundnut seed is nutritionally rich due to presence of lipids, proteins, essential minerals, vitamins, phytosterols and phytochemicals. Consequently, groundnut is playing a vital role as an oilseed as well as food crop (Mondal and Puteh, 2013).

Characterization and identification of plant varieties are fundamental to the development, release and popularization of the crop varieties. Seed is the 'custodian' of genetic improvements in crop species takes place from time through research endeavours in plant breeding. For farmers to realize the full benefits of such improvements, availability of good quality seed is a pre-requisite in crop production. In this context, varietal description for identification of crop varieties has attained a critical importance in national and international seed programmes and there is a considerable need for the development of reliable methods and identifiable characters for identification purpose. The characters for which a variety is distinct from others could be morphological, chemical and biochemical or physiological in nature which aids in varietal identification. The varietal purity is usually tested by heritable characters of seeds, seedlings or growing plants in a field. Grow out test (GOT) is conducted by growing the plants under field condition and growth feature are observed in fixing genuineness. These morphological descriptors have traditional significance and are immediately accessible on the spot without the need of equipments. Distinctive variation in any morphological character, either vegetative or reproductive, could be an aid in the identification of cultivars. Moreover, this help both in management and varietal improvement efforts required for overall increase in crop production. Stem, branch and leaflet characters have been widely used in classifying groundnut cultivars (Ntundu et al. 2006). Mouli and Patil (1976) suggested that the classification of branching types should take into account the presence or absence of primary, secondary and tertiary branches as well as their development. Doku and Asiama (1978) reported variation in shape, size and hairiness between leaflets of the same cultivars, and even on the same plant. Additionally, they also suggested the use of floral characters for identification of plants at specific and higher levels. In groundnut classification, generally both quantitative and qualitative characters of pods have been widely used (Pasquet et al. 1999; Ntundu et al. 2006; Bayor et al. 2010). Further, Multivariate analysis have been used successfully to classify and order variation observed in both quantitative and qualitative traits in the collection of many crop germplasm (Chandran \& Padya, 2000; Lara-Fioreze et al. 2013; Latif et al. 2013). Assessment of vegetative and reproductive traits variability of groundnut using multivariate analyses could provide useful information to allow exploitation of the potential of available germplasm. 
This study was therefore under taken to precisely assess differences in the vegetative and reproductive characters amongst 28 elite mutants and two check cultivars for framing keys of future identifying strategies of groundnut and classify genetic dissimilarity among accessions through cluster analysis.

\section{Materials and methods}

Two field experiments were conducted at the Bangladesh Institute of Nuclear Agriculture (BINA), Mymensingh $\left(24^{0} 75^{\prime} \mathrm{N}\right.$ and $\left.90^{0} 50^{\prime} \mathrm{E}\right)$ during Kharif-I (March-June) seasons of 2017 and 2018. The experiments were carried out under rainfed conditions. The soil of experimental land was sandy loam having a total nitrogen $0.065 \%$, organic matter $1.17 \%$, available phosphorus 18.5 ppm, exchangeable potassium $0.30 \mathrm{meq} / 100 \mathrm{~g}$, sulphur $20 \mathrm{ppm}$ and $\mathrm{pH}$ 6.8. Twenty four established mutants of $\mathrm{M}_{6}$ generation developed at BINA by $150-200 \mathrm{~Gy}$ irradiation, four mutants collected from ICRISAT and two released varieties belonging to Spanish group were studied. Randomized complete block design with three replicates was used in both the experiments. Urea, triple super phosphate and muriate of potash were applied during final land preparation at the rate of 40,150 and $120 \mathrm{~kg} \mathrm{ha}^{-1}$, respectively. Seeds were sown on $15^{\text {th }}$ March, 2017 and $10^{\text {th }}$ March, 2018 with plant spacing of $30 \mathrm{~cm}$ and $15 \mathrm{~cm}$. Unit plot size was $2.1 \mathrm{~m} \times 2 \mathrm{~m}$. Intercultural operations were done as and when necessary for normal plant growth and development.

All the plants of the mutants/cultivars were harvested at a time, 135 and 130 days after sowing for experiment-1 and-2, respectively. Data on different vegetative and reproductive characters were recorded from the experiments following standard methods. For quantitative characters ten plants were scored per accession for each replication. Data were analyzed statistically as per the design used following the analysis of variance (ANOVA) technique and the mean differences were adjusted with DMRT at 5\% level of significance using the statistical computer package program, MSTAT-C following Russell (1986). The morphological data were further analyzed using Euclidian distance coefficient (Zivkovic et al., 2012), NTSYS-pc Versions 2.1 (Rohlf, 2002) program. These similarity coefficient were used to produce a dendrogram for which the UPGMA algorithm and SAHN clustering (unweighted pair group method using arithmetic average) was employed for depicting the genetic relationships. Data were subjected to principal component analysis (PCA) using the same program. The eigenvectors and eigenvalues were determined in PCA. Eigenvectors are the weights in a linear transformation when computing principal component scores while eigenvalues indicate the amount of variance explained by each principal component.

\section{Results and discussion}

\subsection{Vegetative characters}

The effect of season and genotype and their interaction showed highly significant differences for vegetative characters except number of secondary branches for season and leaflet width for interaction of season and genotype (Table 1). This means significant differences existed for season and genotype while some genotypes performed better or worse over a season.

Table 1: Mean Square of Combined Analysis of Variance for Vegetative Characters in 30 Groundnut Genotypes Over Years

\begin{tabular}{|c|c|c|c|c|c|c|c|}
\hline Source of variation & df & $\begin{array}{l}\text { Plant height } \\
(\mathrm{cm})\end{array}$ & $\begin{array}{l}\text { Primary branch } \\
\text { length }(\mathrm{cm})\end{array}$ & $\begin{array}{l}\text { Primary } \\
\text { branches plant } \\
{ }^{1} \text { (no) }\end{array}$ & $\begin{array}{l}\text { Secondary } \\
\text { branches plant }^{-1} \\
\text { (no) }\end{array}$ & $\begin{array}{l}\text { Leaflet length } \\
(\mathrm{cm})\end{array}$ & $\begin{array}{l}\text { Leaflet width } \\
(\mathrm{cm})\end{array}$ \\
\hline Replication & 2 & $21.90 *$ & $26.13^{*}$ & $0.004^{\mathrm{ns}}$ & $0.046^{*}$ & $0.001 \mathrm{~ns}$ & $0.051 * *$ \\
\hline Year (A) & 1 & $325.6^{* *}$ & $625.7 * *$ & $36.74 * *$ & $0.018^{\mathrm{ns}}$ & $0.312 * *$ & $0.057 * *$ \\
\hline Genotype (B) & 29 & $422.9 * *$ & $478.2 * *$ & $5.62 * *$ & $34.23^{* *}$ & $0.948 * *$ & $0.240 * *$ \\
\hline $\mathrm{A} \times \mathrm{B}$ & 29 & $24.15^{* * *}$ & $25.38 * *$ & $2.68 * *$ & $1.22 * *$ & $0.028 * *$ & $0.004^{\mathrm{ns}}$ \\
\hline Error & 118 & 7.33 & 9.18 & 0.356 & 0.010 & 0.012 & 0.005 \\
\hline
\end{tabular}

*,**: Indicates Significant at 5\%, And 1\% Level of Probability, Respectively.

Table 2: Mean of Different Vegetative Characters Amongst 30 Mutants and Cultivars Averaged Over Two Experiments

\begin{tabular}{|c|c|c|c|c|c|c|}
\hline Mutants/ cultivars & $\begin{array}{l}\text { Plant height } \\
(\mathrm{cm})\end{array}$ & $\begin{array}{l}\text { Cotyledonary } \\
\text { branch length }(\mathrm{cm})\end{array}$ & $\begin{array}{l}\text { Primary branches } \\
\text { plant }^{-1} \text { (no) }\end{array}$ & $\begin{array}{l}\text { Secondary } \\
\text { branches plant }{ }^{-1} \\
\text { (no) }\end{array}$ & $\begin{array}{l}\text { Leaflet length } \\
(\mathrm{cm})\end{array}$ & $\begin{array}{l}\text { Leaflet width } \\
(\mathrm{cm})\end{array}$ \\
\hline Mut-3 & $32.3 \mathrm{~h}$ & $59.0 \mathrm{~b}$ & $7.1 \mathrm{ab}$ & 0 & $3.70 \mathrm{f}$ & $1.80 \mathrm{f}$ \\
\hline $\mathrm{D}_{1} / 23-73$ & $48.7 \mathrm{a}$ & $59.0 \mathrm{~b}$ & $6.2 \mathrm{bc}$ & 0 & $4.24 \mathrm{c}$ & $2.09 \mathrm{~b}$ \\
\hline Mut-6 & $42.7 \mathrm{c}$ & $46.7 \mathrm{f}$ & $5.0 \mathrm{~d}$ & 0 & $3.95 \mathrm{e}$ & $1.98 \mathrm{c}$ \\
\hline $\mathrm{D}_{1} / 62-30$ & $51.3 \mathrm{a}$ & $59.0 \mathrm{~b}$ & $5.0 \mathrm{~d}$ & $6.0 \mathrm{~b}$ & $4.10 \mathrm{~d}$ & $1.86 \mathrm{e}$ \\
\hline $\mathrm{M}_{6} / 7-25$ & $31.3 \mathrm{hi}$ & $43.3 \mathrm{f}$ & $8.0 \mathrm{a}$ & $1.2 \mathrm{~g}$ & $3.61 \mathrm{~g}$ & $1.77 \mathrm{f}$ \\
\hline $\mathrm{D}_{1} / 43-17$ & $52.3 \mathrm{a}$ & $52.3 \mathrm{~d}$ & $5.3 \mathrm{~d}$ & 0 & $3.95 \mathrm{c}$ & $1.87 \mathrm{e}$ \\
\hline $\mathrm{M}_{6} / 48-46$ & $39.8 \mathrm{~d}$ & $56.3 \mathrm{~b}$ & $5.0 \mathrm{~d}$ & 0 & $3.92 \mathrm{c}$ & $1.92 \mathrm{~d}$ \\
\hline$M_{6} / 60-37$ & $44.2 \mathrm{~b}$ & $57.3 \mathrm{~b}$ & $6.1 \mathrm{bc}$ & $3.9 \mathrm{c}$ & $4.11 \mathrm{~d}$ & $1.96 \mathrm{c}$ \\
\hline $\mathrm{D}_{1} / 82-65$ & $37.0 \mathrm{f}$ & $43.3 \mathrm{f}$ & $5.1 \mathrm{~d}$ & $4.0 \mathrm{c}$ & $4.09 \mathrm{~d}$ & $1.98 \mathrm{c}$ \\
\hline $\mathrm{M}_{6} / 70-19$ & $39.7 \mathrm{~d}$ & $47.0 \mathrm{f}$ & $5.5 \mathrm{~d}$ & $0.1 \mathrm{~h}$ & $3.60 \mathrm{~g}$ & $1.66 \mathrm{~g}$ \\
\hline $\mathrm{M}_{6} / 90-39$ & 32.7 hi & $42.0 \mathrm{~h}$ & $6.0 \mathrm{bc}$ & 0 & $3.57 \mathrm{~h}$ & $1.58 \mathrm{i}$ \\
\hline $\mathrm{M}_{6} / 58-18$ & $43.8 \mathrm{~b}$ & $46.0 \mathrm{f}$ & $5.0 \mathrm{~d}$ & 0 & $3.71 \mathrm{f}$ & $1.82 \mathrm{e}$ \\
\hline $\mathrm{D}_{1} / 36-2$ & $43.0 \mathrm{c}$ & $45.0 \mathrm{f}$ & $5.0 \mathrm{~d}$ & 0 & $3.96 \mathrm{e}$ & $1.90 \mathrm{e}$ \\
\hline $\mathrm{M}_{6} / 39-11$ & $32.2 \mathrm{hi}$ & $36.3 \mathrm{i}$ & $6.3 \mathrm{bc}$ & 0 & $3.72 \mathrm{f}$ & $1.70 \mathrm{~g}$ \\
\hline $\mathrm{M}_{6} / 79-71$ & $39.7 \mathrm{~d}$ & $45.7 \mathrm{f}$ & $5.9 \mathrm{~b}$ & 0 & $3.47 \mathrm{i}$ & $1.78 \mathrm{f}$ \\
\hline $\mathrm{M}_{6} / 40-34$ & $38.3 \mathrm{e}$ & $37.7 \mathrm{e}$ & $7.0 \mathrm{ab}$ & $2.1 \mathrm{f}$ & $3.51 \mathrm{~h}$ & $1.67 \mathrm{~g}$ \\
\hline $\mathrm{D}_{1} / 24-29$ & $49.3 \mathrm{a}$ & $77.3 \mathrm{a}$ & $5.2 \mathrm{~d}$ & $10.0 \mathrm{a}$ & $4.42 \mathrm{~b}$ & $2.00 \mathrm{c}$ \\
\hline $\mathrm{M}_{6} / 36-24$ & $42.8 \mathrm{c}$ & $48.0 \mathrm{e}$ & $4.9 \mathrm{~d}$ & 0 & $4.05 \mathrm{~d}$ & $2.00 \mathrm{c}$ \\
\hline $\mathrm{M}_{6} / 16-90$ & $28.8 \mathrm{jk}$ & $43.3 \mathrm{f}$ & $5.0 \mathrm{~d}$ & 0 & $3.05 \mathrm{k}$ & $1.78 \mathrm{f}$ \\
\hline $\mathrm{M}_{6} / 64-82$ & $26.0 \mathrm{k}$ & $36.0 \mathrm{i}$ & $4.8 \mathrm{~d}$ & 0 & $3.39 \mathrm{j}$ & $1.70 \mathrm{~g}$ \\
\hline $\mathrm{D}_{1} / 11-60$ & $52.7 \mathrm{a}$ & $59.0 \mathrm{~b}$ & $6.1 \mathrm{bc}$ & $3.0 \mathrm{c}$ & $4.59 \mathrm{a}$ & $2.06 \mathrm{~b}$ \\
\hline$M_{6} / 57-15$ & $25.8 \mathrm{k}$ & $47.7 \mathrm{f}$ & $3.5 \mathrm{ef}$ & $0.9 \mathrm{~g}$ & $3.57 \mathrm{~h}$ & $1.96 \mathrm{c}$ \\
\hline $\mathrm{D}_{1} / 86-54$ & $48.0 \mathrm{a}$ & $48.3 \mathrm{e}$ & $5.0 \mathrm{~d}$ & $4.0 \mathrm{c}$ & $4.42 \mathrm{~b}$ & $2.18 \mathrm{a}$ \\
\hline $\mathrm{D}_{1} / 28-38$ & $44.8 \mathrm{~b}$ & $47.7 \mathrm{f}$ & $4.9 \mathrm{~d}$ & $2.2 \mathrm{f}$ & $4.04 \mathrm{~d}$ & $1.94 \mathrm{~d}$ \\
\hline Mut-2 & $34.0 \mathrm{~g}$ & $42.7 \mathrm{~g}$ & $3.0 \mathrm{ef}$ & $3.0 \mathrm{~d}$ & $3.45 \mathrm{i}$ & $1.74 \mathrm{~g}$ \\
\hline
\end{tabular}




\begin{tabular}{lllllll}
\hline $\mathrm{M}_{6} / 61-6$ & $29.0 \mathrm{jk}$ & $47.0 \mathrm{f}$ & $4.0 \mathrm{e}$ & 0 & $4.26 \mathrm{c}$ \\
Mut-5 $_{\mathrm{M}_{6} / 54-20}$ & $28.3 \mathrm{jk}$ & $36.0 \mathrm{i}$ & $5.3 \mathrm{~d}$ & $5.0 \mathrm{~d}$ & 0 & $3.44 \mathrm{i}$ \\
Dhaka-1 & $31.2 \mathrm{ij}$ & $35.0 \mathrm{i}$ & $7.2 \mathrm{ab}$ & 0 & $3.00 \mathrm{k}$ \\
Zhingabadam & $47.3 \mathrm{~b}$ & $42.3 \mathrm{~h}$ & $5.7 \mathrm{~d}$ & 0 & $4.67 \mathrm{a}$ \\
Range & $52.5 \mathrm{a}$ & $53.7 \mathrm{e}$ & $4.0-8.0$ & $0-10$ & $3.86 \mathrm{e}$ \\
CV $(\%)$ & $25.8-52.7$ & $35.0-67.3$ & 10.62 & 26.02 & $3.07-4.67$ \\
\hline
\end{tabular}

Figures bearing the same letter (s) within a column do not differ significantly at $\mathrm{P} \leq 0.05$ by DMRT.

The mean effects of genotype on vegetative characters are shown in the Table 2 . The vegetative characters showed significant differences amongst the mutants and the check cultivars (Table 2). Plant height (main stem height) showed a wide range of variability, with mutants $\mathrm{D}_{1} / 11-60(52.7 \mathrm{~cm})$ and $\mathrm{M}_{6} / 57-15(25.8 \mathrm{~cm})$ being the tallest and shortest of all, respectively. In contrast, five mutants $\mathrm{D}_{1} / 23-73, \mathrm{D}_{1} / 62-$ $30, \mathrm{D}_{1} / 43-17, \mathrm{D}_{1} / 24-49$ and $\mathrm{D}_{1} / 86-54$, and one cultivar Zhingabadam showed non-significant differences from the tallest group whilst the four others namely, $M_{6} / 16-90, M_{6} / 64-82, M_{6} / 61-6$ and Mut-5 were at par with the shortest one. The remainder formed an intermediate group.

Cotyledonary branch length had shown considerable variation ranging from 35.0 to $77.3 \mathrm{~cm}$. Mutant $\mathrm{D}_{1} / 24-29$ had the longest cotyledonary branch $\left(77.3 \mathrm{~cm}\right.$ ) with a significant difference from the rest (Table 2). In contrast, mutant $\mathrm{D}_{6} / 54-20$ showed the shortest cotyledonary branch $(35.0 \mathrm{~cm})$ and was not significantly different from $\mathrm{M}_{6} / 39-11, \mathrm{M}_{6} / 64-82$ and Mut-5. Mutant $\mathrm{M}_{6} / 7-25$ had the highest number of primary branches $\left(8\right.$ plant $\left.^{-1}\right)$ and was statistically at par with its parent, Dhaka-1 (7.2 plant $\left.{ }^{-1}\right)$. In contrast, mutant Mut-2 had the lowest number of primary branches (3.0 plant ${ }^{-1}$ ) followed by M6/57-15 (3.5 plant $^{-1}$ ) with same statistical rank. The mutants $\mathrm{M}_{1} / 24-29$ and $\mathrm{M}_{1} / 62-$ 30 produced the highest $\left(10\right.$ plant $\left.^{-1}\right)$ and second highest $\left(6\right.$ plant $\left.^{-1}\right)$ secondary branches and showed significant differences from each other and all other mutants. In contrast, 17 mutants/varieties had no secondary branches.

Leaflet length and breadth are central attributes of leaf sizes and shapes which showed the least variation (Table 2). The leaflet length and breadth of Dhaka-1 was the highest with that of mutant $\mathrm{M}_{6} / 54-20$ being the lowest, and these two were significantly different from the remainder. The above results of variability are in full conformity with many workers (Patil, 1972; Mouli and Patil, 1976; Bayor et al. 2010).

\subsection{Reproductive characters}

Season had no great influence on reproductive characters while genotypes had great effect on reproductive characters (Table 3 ). It means reproductive characters are not greatly influenced by season like vegetative characters.

Table 3: Mean Square of Combined Analysis of Variance for Reproductive Characters in 30 Groundnut Genotypes Over Years

\begin{tabular}{|c|c|c|c|c|c|c|c|c|c|}
\hline Source of variation & df & $\begin{array}{l}\text { Buds/ ra- } \\
\text { ceme (no) }\end{array}$ & $\begin{array}{l}\text { Calyx tube } \\
\text { length }(\mathrm{cm})\end{array}$ & $\begin{array}{l}\text { Std petal } \\
\text { length }(\mathrm{cm})\end{array}$ & $\begin{array}{l}\text { Std petal } \\
\text { breadth } \\
(\mathrm{cm})\end{array}$ & $\begin{array}{l}\text { Pod length } \\
(\mathrm{cm})\end{array}$ & $\begin{array}{l}\text { Pod } \\
\text { breadth } \\
(\mathrm{cm})\end{array}$ & $\begin{array}{l}\text { 100-pod } \\
\text { weight (g) }\end{array}$ & $\begin{array}{l}100 \text { - kernel } \\
\text { weight }(\mathrm{g})\end{array}$ \\
\hline Replication & 2 & $0.00 * *$ & $0.054^{\mathrm{ns}}$ & $0.001^{\mathrm{ns}}$ & $0.039 * *$ & $0.005^{\mathrm{ns}}$ & $0.001^{\mathrm{ns}}$ & $9.33^{\mathrm{ns}}$ & $20.33^{*}$ \\
\hline Year (A) & 1 & $0.00^{\mathrm{ns}}$ & $0.176^{*}$ & $0.039 *$ & $0.014^{\mathrm{ns}}$ & $0.026^{\mathrm{ns}}$ & $0.014^{\mathrm{ns}}$ & $36.6^{*}$ & $90.2 *$ \\
\hline Genotype (B) & 29 & $0.39 * *$ & $1.855^{* *}$ & $0.116^{* *}$ & $0.131 * *$ & $0.315 * *$ & $0.111 * *$ & $5711 * *$ & $1065^{* *}$ \\
\hline $\mathrm{A} \times \mathrm{B}$ & 29 & $0.00^{\mathrm{ns}}$ & $0.012^{\mathrm{ns}}$ & $0.003^{\mathrm{ns}}$ & $0.003^{\mathrm{ns}}$ & $0.004^{\mathrm{ns}}$ & $0.002^{\mathrm{ns}}$ & $18.22 *$ & $8.01 *$ \\
\hline Error & 118 & 0.000 & 0.022 & 0.004 & 0.005 & 0.007 & 0.004 & 9.838 & 3.838 \\
\hline
\end{tabular}

*,**: Indicates significant at 5\%, and $1 \%$ level of probability, respectively; Std: Standard

The interaction effect of genotype and season also had no significant differences in most of the reproductive characters except 100-pod and 100-kernel weight. The mean effects of genotype on reproductive characters are shown in the Table 3.

Among different flower characters, buds per raceme had the highest variability followed by calyx tube length, standard petal length and breadth (Table 4). Most of the mutants and cultivars were alike in showing single bud per raceme, excepting $\mathrm{M}_{6} / 36-24$ and $\mathrm{D}_{6} / 61-6$ that showed more than one bud. Calyx tube length was the highest in Mut-5 $(5.05 \mathrm{~cm})$ followed by Mut-6 $(4.47 \mathrm{~cm})$ and $\mathrm{D}_{1} / 36-2(4.33 \mathrm{~cm})$.

Table 4: Mean of Different Reproductive Characters Amongst 30 Mutants and Cultivars Averaged Over Two Experiments

\begin{tabular}{|c|c|c|c|c|c|c|c|c|}
\hline $\begin{array}{l}\text { Mutants/ } \\
\text { cultivars }\end{array}$ & $\begin{array}{l}\text { Buds ra- } \\
\text { ceme }^{-1} \text { (no) }\end{array}$ & $\begin{array}{l}\text { Calyx tube } \\
\text { length }(\mathrm{cm})\end{array}$ & $\begin{array}{l}\text { Standard petal } \\
\text { length }(\mathrm{cm})\end{array}$ & $\begin{array}{l}\text { Standard petal } \\
\text { breadth }(\mathrm{cm})\end{array}$ & $\begin{array}{l}\text { Pod length } \\
(\mathrm{cm})\end{array}$ & $\begin{array}{l}\text { Pod breadth } \\
(\mathrm{cm})\end{array}$ & $\begin{array}{l}\text { 100-pod } \\
\text { weight (g) }\end{array}$ & $\begin{array}{l}\text { 100- kernel } \\
\text { weight }(\mathrm{g})\end{array}$ \\
\hline Mut-3 & $1 \mathrm{~b}$ & $3.70 \mathrm{e}$ & $1.37 \mathrm{e}$ & $1.75 \mathrm{~b}$ & $2.62 \mathrm{~b}$ & $1.44 \mathrm{a}$ & $186.5 \mathrm{a}$ & $67.67 \mathrm{c}$ \\
\hline $\mathrm{D}_{1} / 23-73$ & $1 \mathrm{~b}$ & $3.53 \mathrm{f}$ & $1.13 \mathrm{~d}$ & $1.37 \mathrm{~h}$ & $2.13 \mathrm{e}$ & $1.08 \mathrm{~d}$ & $83.83 \mathrm{j}$ & $33.33 \mathrm{~m}$ \\
\hline Mut-6 & $1 \mathrm{~b}$ & $4.47 \mathrm{~b}$ & $1.20 \mathrm{c}$ & $1.55 \mathrm{f}$ & $2.47 \mathrm{c}$ & $1.30 \mathrm{~b}$ & $103.5 \mathrm{e}$ & $47.67 \mathrm{~d}$ \\
\hline $\mathrm{D}_{1} / 62-30$ & $1 \mathrm{~b}$ & $3.17 \mathrm{~h}$ & $1.07 \mathrm{e}$ & $1.60 \mathrm{e}$ & $2.20 \mathrm{~d}$ & $1.20 \mathrm{c}$ & $95.27 \mathrm{f}$ & $37.67 \mathrm{~h}$ \\
\hline $\mathrm{M}_{6} / 7-25$ & $1 \mathrm{~b}$ & $3.73 \mathrm{e}$ & $1.32 \mathrm{~b}$ & $1.73 \mathrm{~b}$ & $2.20 \mathrm{~d}$ & $1.13 \mathrm{~d}$ & $101.4 \mathrm{e}$ & $43.00 \mathrm{e}$ \\
\hline $\mathrm{D}_{1} / 43-17$ & $1 \mathrm{~b}$ & $3.73 \mathrm{e}$ & $1.13 \mathrm{~d}$ & $1.53 \mathrm{~g}$ & $2.11 \mathrm{e}$ & $1.07 \mathrm{~d}$ & $82.33 \mathrm{k}$ & $35.33 \mathrm{j}$ \\
\hline $\mathrm{M}_{6} / 48-46$ & $1 \mathrm{~b}$ & $3.47 \mathrm{f}$ & $1.08 \mathrm{e}$ & $1.60 \mathrm{e}$ & $1.98 \mathrm{f}$ & $1.17 \mathrm{c}$ & $75.03 \mathrm{n}$ & $34.00 \mathrm{n}$ \\
\hline$M_{6} / 60-37$ & $1 \mathrm{~b}$ & $2.67 \mathrm{k}$ & $1.23 \mathrm{c}$ & $1.50 \mathrm{~g}$ & $2.16 \mathrm{e}$ & $1.24 \mathrm{c}$ & $87.60 \mathrm{i}$ & $43.33 \mathrm{e}$ \\
\hline $\mathrm{D}_{1} / 82-65$ & $1 \mathrm{~b}$ & $3.70 \mathrm{e}$ & $1.30 \mathrm{c}$ & $1.65 \mathrm{c}$ & $2.08 \mathrm{f}$ & $1.03 \mathrm{~d}$ & $89.60 n$ & $35.33 \mathrm{e}$ \\
\hline $\mathbf{M}_{6} / 70-19$ & $1 \mathrm{~b}$ & $3.00 \mathrm{i}$ & $1.40 \mathrm{~b}$ & $1.70 \mathrm{~b}$ & $2.19 \mathrm{e}$ & $1.22 \mathrm{e}$ & $86.93 \mathrm{i}$ & $38.00 \mathrm{j}$ \\
\hline $\mathrm{M}_{6} / 90-39$ & $1 \mathrm{~b}$ & $2.60 \mathrm{k}$ & $1.10 \mathrm{~d}$ & $1.50 \mathrm{~g}$ & $2.29 \mathrm{~d}$ & $1.14 \mathrm{c}$ & 79.031 & $41.67 \mathrm{~h}$ \\
\hline $\mathrm{M}_{6} / 58-18$ & $1 \mathrm{~b}$ & $3.37 \mathrm{~g}$ & $1.16 \mathrm{~d}$ & $1.57 \mathrm{f}$ & $2.19 \mathrm{e}$ & $1.15 \mathrm{c}$ & $98.40 \mathrm{e}$ & $39.33 \mathrm{f}$ \\
\hline $\mathrm{D}_{1} / 36-2$ & $1 \mathrm{~b}$ & $4.33 \mathrm{~b}$ & $1.23 \mathrm{c}$ & $1.63 \mathrm{~d}$ & $2.18 \mathrm{e}$ & $1.07 \mathrm{~d}$ & $81.23 \mathrm{k}$ & $36.67 \mathrm{i}$ \\
\hline $\mathrm{M}_{6} / 39-11$ & $1 \mathrm{~b}$ & $3.83 \mathrm{e}$ & $1.27 \mathrm{c}$ & $1.50 \mathrm{~g}$ & $2.26 \mathrm{~d}$ & $1.17 \mathrm{c}$ & $100.9 \mathrm{c}$ & $40.33 \mathrm{f}$ \\
\hline $\mathrm{M}_{6} / 79-71$ & $1 \mathrm{~b}$ & $3.07 \mathrm{i}$ & $1.32 \mathrm{~b}$ & $1.67 \mathrm{~b}$ & $2.56 \mathrm{~b}$ & $1.43 \mathrm{a}$ & $134.4 \mathrm{c}$ & $66.33 \mathrm{c}$ \\
\hline $\mathrm{M}_{6} / 40-34$ & $1 \mathrm{~b}$ & $3.47 \mathrm{f}$ & $1.30 \mathrm{c}$ & $1.72 \mathrm{~b}$ & $2.24 \mathrm{~d}$ & $1.12 \mathrm{~d}$ & $93.33 \mathrm{~g}$ & $40.67 \mathrm{f}$ \\
\hline $\mathrm{D}_{1} / 24-29$ & $1 \mathrm{~b}$ & $3.30 \mathrm{~g}$ & $1.50 \mathrm{a}$ & $1.20 \mathrm{j}$ & $1.98 \mathrm{f}$ & $1.05 \mathrm{~d}$ & $75.97 \mathrm{n}$ & $28.67 \mathrm{o}$ \\
\hline$M_{6} / 36-24$ & $2 a$ & $3.83 \mathrm{e}$ & $1.20 \mathrm{c}$ & $1.53 \mathrm{~g}$ & $2.12 \mathrm{e}$ & $1.06 \mathrm{~d}$ & 77.531 & 33.671 \\
\hline $\mathrm{M}_{6} / 16-90$ & $1 \mathrm{~b}$ & $3.10 \mathrm{~h}$ & $1.31 \mathrm{c}$ & $1.67 \mathrm{~b}$ & $2.23 \mathrm{~d}$ & $1.16 \mathrm{c}$ & $91.47 \mathrm{~h}$ & $39.33 \mathrm{~g}$ \\
\hline$M_{6} / 64-82$ & $1 \mathrm{~b}$ & $3.53 \mathrm{f}$ & $1.17 \mathrm{~d}$ & $1.67 \mathrm{~b}$ & $2.26 \mathrm{~d}$ & $1.17 \mathrm{c}$ & 86.77 i & $46.00 \mathrm{~d}$ \\
\hline $\mathrm{D}_{1} / 11-60$ & $1 \mathrm{~b}$ & $3.53 \mathrm{f}$ & $1.02 \mathrm{e}$ & $1.32 \mathrm{i}$ & $2.06 \mathrm{f}$ & $1.03 \mathrm{~d}$ & $76.83 \mathrm{~m}$ & $32.33 \mathrm{n}$ \\
\hline$M_{6} / 57-15$ & $1 \mathrm{~b}$ & $4.03 \mathrm{c}$ & $1.34 \mathrm{~b}$ & $1.67 \mathrm{~b}$ & $2.29 \mathrm{~d}$ & $1.15 \mathrm{c}$ & $92.90 \mathrm{~g}$ & $43.67 \mathrm{e}$ \\
\hline $\mathrm{D}_{1} / 86-54$ & $1 \mathrm{~b}$ & $2.61 \mathrm{k}$ & $1.10 \mathrm{~d}$ & $1.50 \mathrm{~g}$ & $2.02 \mathrm{f}$ & $1.03 \mathrm{~d}$ & 79.401 & $32.67 \mathrm{~m}$ \\
\hline $\mathrm{D}_{1} / 28-38$ & $1 \mathrm{~b}$ & $2.90 \mathrm{j}$ & $1.20 \mathrm{c}$ & $1.50 \mathrm{~g}$ & $2.14 \mathrm{c}$ & $1.08 \mathrm{~d}$ & $81.87 \mathrm{k}$ & $34.33 \mathrm{k}$ \\
\hline Mut-2 & $1 \mathrm{~b}$ & $3.90 \mathrm{~d}$ & $1.59 \mathrm{a}$ & $1.90 \mathrm{a}$ & $2.87 \mathrm{a}$ & $1.52 \mathrm{a}$ & $190.2 \mathrm{a}$ & $81.67 \mathrm{a}$ \\
\hline
\end{tabular}




\begin{tabular}{|c|c|c|c|c|c|c|c|c|}
\hline$M_{6} / 61-6$ & $2 \mathrm{a}$ & $3.40 \mathrm{~g}$ & $1.25 \mathrm{c}$ & $1.77 \mathrm{~b}$ & $2.06 \mathrm{f}$ & $1.07 \mathrm{~d}$ & $71.73 \mathrm{o}$ & 33.671 \\
\hline Mut-5 & $1 \mathrm{~b}$ & $5.04 \mathrm{a}$ & $1.53 \mathrm{a}$ & $1.85 \mathrm{a}$ & $2.48 \mathrm{c}$ & $1.43 \mathrm{a}$ & $162.4 \mathrm{~b}$ & $76.67 \mathrm{~b}$ \\
\hline $\mathrm{M}_{6} / 54-20$ & $1 \mathrm{~b}$ & $3.10 \mathrm{~h}$ & $1.42 \mathrm{~b}$ & $1.67 \mathrm{~b}$ & $2.40 \mathrm{c}$ & $1.35 \mathrm{~b}$ & $92.62 \mathrm{~g}$ & $40.00 \mathrm{f}$ \\
\hline Dhaka-1 & $1 \mathrm{~b}$ & $3.57 \mathrm{f}$ & $1.27 \mathrm{c}$ & $1.63 \mathrm{c}$ & $2.05 \mathrm{f}$ & $1.16 \mathrm{c}$ & $71.30 \mathrm{o}$ & $31.33 \mathrm{n}$ \\
\hline $\begin{array}{l}\text { Zhingaba- } \\
\text { dam }\end{array}$ & $1 \mathrm{~b}$ & $2.61 \mathrm{k}$ & $1.33 \mathrm{~b}$ & $1.68 \mathrm{~b}$ & $2.84 \mathrm{a}$ & $1.32 \mathrm{~b}$ & $119.7 \mathrm{~d}$ & $30.67 \mathrm{o}$ \\
\hline Range & $1-2$ & $2.63-5.04$ & $1.02-1.59$ & $1.20-1.90$ & $1.98-2.87$ & $1.03-1.52$ & 71.3-190.2 & $30.67-81.67$ \\
\hline $\mathrm{CV}(\%)$ & 13.8 & 9.85 & 5.04 & 2.86 & 2.42 & 5.09 & 4.69 & 5.19 \\
\hline
\end{tabular}

Figures bearing the same letter (s) within a column do not differ significantly at $\mathrm{P} \leq 0.05$ by DMRT.

In contrast, Zhingabadam had the lowest tube length $(2.61 \mathrm{~cm})$ and shared equal rank with four other mutants (range $2.63-2.70 \mathrm{~cm}$ ). Standard petal length, in general, appeared lower than their breadth amongst the mutants and cultivars. Standard petal length was the highest in Mut-2 $(1.59 \mathrm{~cm})$ and showed insignificant difference with Mut-5 $(1.53 \mathrm{~cm})$ and $\mathrm{D}_{1} / 24-29(1.50 \mathrm{~cm})$. Contrarily, mutant $\mathrm{D}_{1} / 11$ 60 was the shortest of all in standard petal length $(1.02 \mathrm{~cm})$. Once again, Mut-2 showed the highest standard petal breadth $(1.90 \mathrm{~cm})$ while that of $D_{1} / 24-29$ was the lowest $(1.20 \mathrm{~cm})$. Patil and Mouli (1977) observed high variability in calyx tube and standard petal length and breadth of groundnut.

Mut-2 had the highest pod length $(2.87 \mathrm{~cm})$ but identical with Zhingabadam $(2.84 \mathrm{~cm})$ while mutants $\mathrm{D}_{1} / 24-29$ and $\mathrm{M}_{6} / 48-46 \mathrm{had}$ the lowest $(1.98 \mathrm{~cm})$ and insignificant different with other five mutants/cultivar D $1 / 82-65$, D $1 / 11-60, \mathrm{D}_{1} / 86-54, \mathrm{M}_{6} / 61-6$ and Dhaka-1 (Table 4). Once again, Mut-2 had the highest pod breadth $(1.52 \mathrm{~cm})$ but at par with Mut-3 $(1.44 \mathrm{~cm})$, Mut-5 $(1.43 \mathrm{~cm})$ and M6/79-71 (1.43 cm). In general, those mutants that showed the lowest pod length also showed the lowest pod breadth. Kernel and pod weights mostly displayed significant variability amongst the mutants and cultivars. Those mutants which showed higher pod weights also showed higher kernel weights. The highest 100-pod (190.2 g) and 100-kernel (81.67 g) weights were recorded in Mut-2. In contrast, Dhaka-1 showed the lowest pod and kernel weights. All the developed mutants have shown extreme variability which are in full conformity with Ashri and Levy (1979) who had observed similar results using chemical mutagens in groundnut.

\subsection{Cluster analysis}

Based on genetic distance, the variation among the groundnut mutant lines ranged from 130.8 to 4.7 (Fig. 1). The highest genetic distance (130.8) was computed between Mut-2 and $\mathrm{D}_{1} / 24-29$ followed by between Mut-2 and $\mathrm{D}_{1} / 11-60$ (126.1) and between Mut-2 and M6/60-37, and the lowest distance (4.7) was observed between $\mathrm{D}_{1} / 28-38$ and $\mathrm{D}_{1} / 36-2$ followed by between $\mathrm{D}_{1} / 28-38$ and $\mathrm{D}_{1} / 86-54$ (4.8) and between $\mathrm{D}_{1} / 28-38$ and $\mathrm{M}_{6} / 36-24$ (5.4). The details of distance matrix are shown in the Supplementary Table 1. Genotypes with higher genetic distances could be used as parents in hybridisations to obtain maximum heterosis as well as to use identifying keys for maintain purity of a genotype. Several authors previously described similar results (Upadhyaya, 2003; Ntundu et al. 2006; Latif et al. 2011).

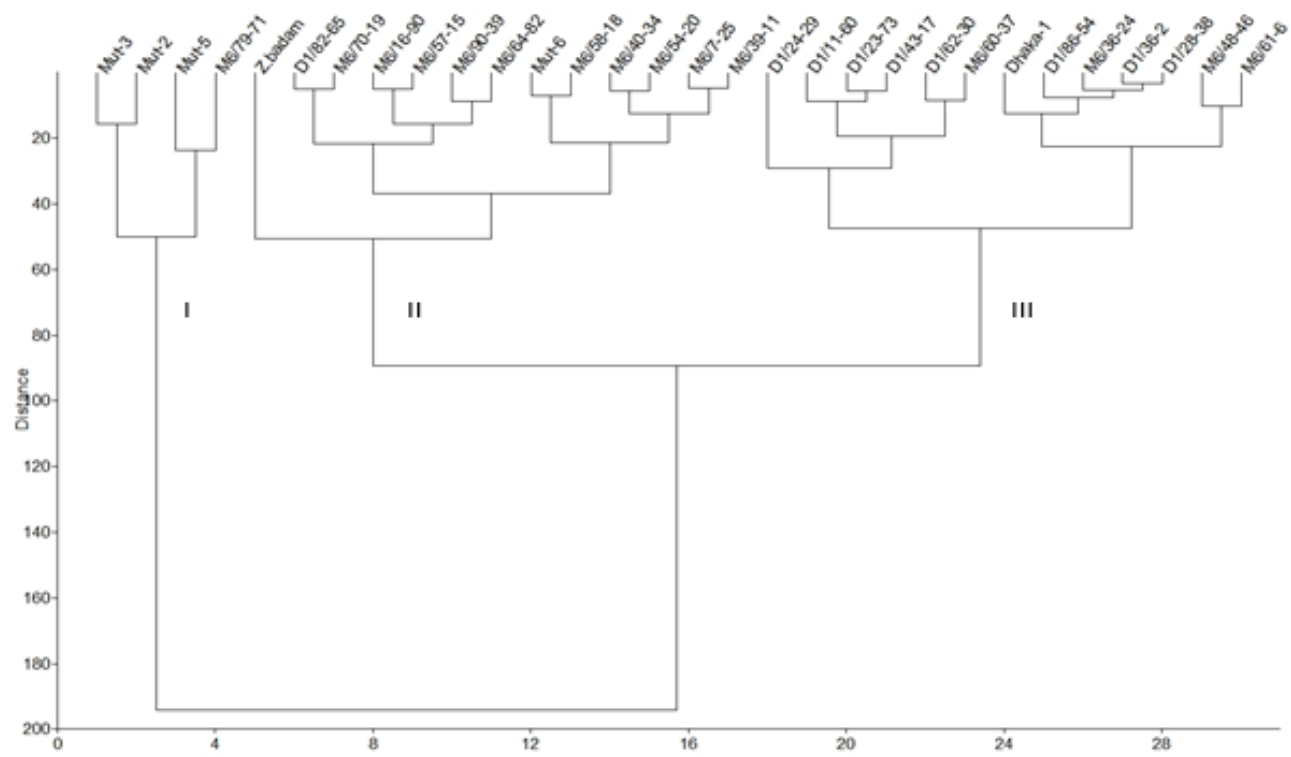

Fig. 1: The Dendrogram Showing Relationship Among 30 Groundnut Genotypes Using 14 Vegetative and Reproductive Traits.

A cluster analysis with genetic distance produced by UPGMA dendrogram (Fig. 1), which clarified the overall genetic relationship between groundnut mutant lines. Based on the genetic distance, the 30 mutant lines were grouped into three major clusters at distance level 60. Cluster I consisted of 4 accessions, cluster II consisted of 13 accessions and cluster III consisted of 13 accessions. The UPGMA dendrogram, based on genetic distances, indicated that mutant lines derived from same parental lines could be classified into same cluster with parent or different clusters.

\subsection{Principal component analysis (PCA)}

Three principal components (PCs) accounted for $98.12 \%$ of the total variation in the 30 mutant lines of groundnut; of these, the first three PCs exhibited variations of $86.21,9.57$ and $2.33 \%$. The characters, 100-pod weight had the highest contribution followed by branch length, plant height and 100-kernel weight (Fig. 2). 


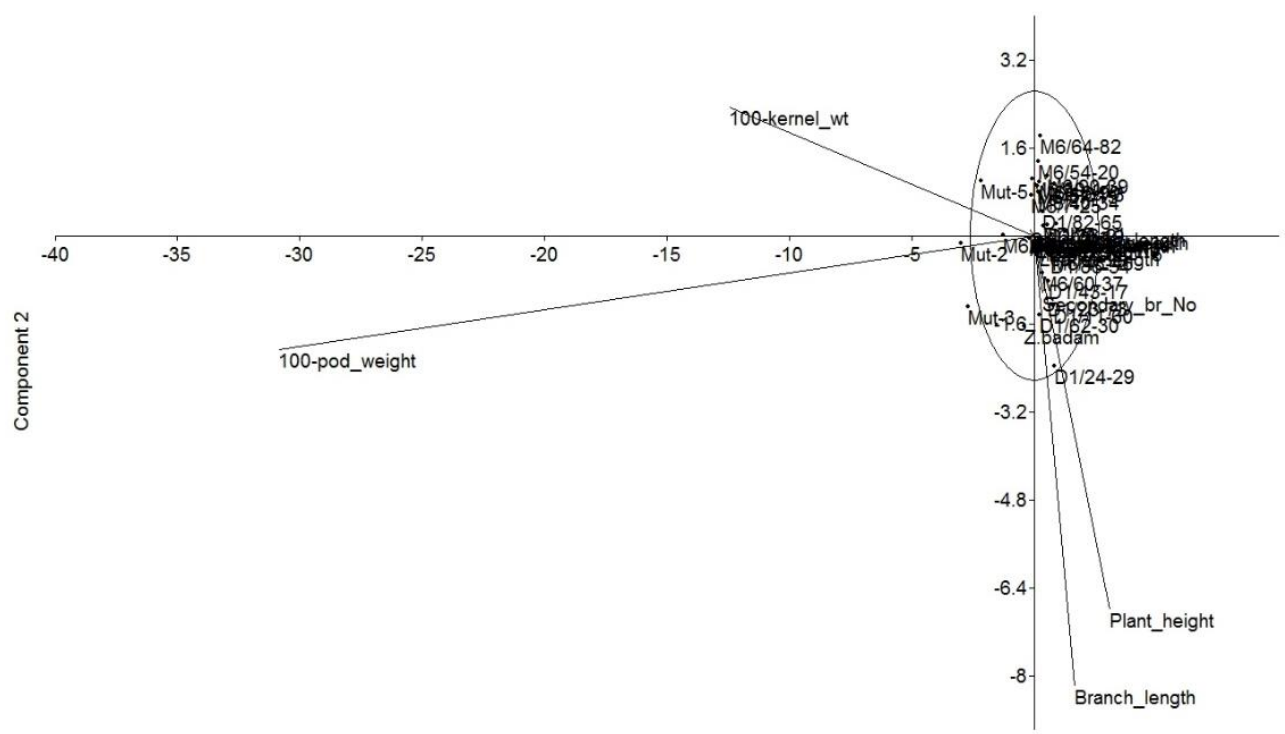

Component 1

Fig. 2: PCA Graph Showing Relationships Among 30 Groundnut Genotypes Using 14 Vegetative and Reproductive Traits.

Several authors have used PCA to determine distinct variations in different crops (Upadhyaya, 2003; Rafii et al. 2012; Latif et al. 2013).

\subsection{Keys for identification}

The keys for identification of mutants and check cultivars based on the vegetative and reproductive structures are shown in Table 5 following Gibbons et al. (1972). The highest number of mutant genotypes was placed in the intermediate scales in any of the key identifying traits, whilst only few in the highest and lowest scale extremes. The latter extremes are actually very important keys in distinguishing the genotypes.

Table 5: Keys to Identification of Groundnut Mutants and Cultivars (Following Gibbons Et Al. 1972)

\begin{tabular}{|c|c|c|}
\hline \multicolumn{2}{|c|}{ Identifying characters } & Name of genotypes \\
\hline \multicolumn{3}{|c|}{ Vegetative characters } \\
\hline \multirow[t]{2}{*}{ I } & a. Plant tall $(\geq 50 \mathrm{~cm})$ & $D_{1} / 62-30, D_{1} / 43-17, D_{1} / 11-60$, Zhingabadam \\
\hline & b. Plant distinctly short $(\leq 26 \mathrm{~cm})$ & $\mathrm{M}_{6} / 64-82, \mathrm{M}_{6} / 57-15$ \\
\hline \multirow[t]{2}{*}{ II } & a. Primary branch distinctly long $(77.3 \mathrm{~cm})$ & $\mathrm{D}_{1} / 24-29$ \\
\hline & b. Primary branch distinctly short $(\leq 37 \mathrm{~cm})$ & $\mathrm{M}_{6} / 39-11, \mathrm{M}_{6} / 64-82, \mathrm{M}_{6} / 54-20$, Mut-5 \\
\hline \multirow[t]{2}{*}{ III } & a. Primary branch number distinctly high $\left(8\right.$ plant $\left.^{-1}\right)$ & $\mathrm{M}_{6} / 7-25$ \\
\hline & b. Primary branch number less $\left(3\right.$ plant $\left.^{-1}\right)$ & Mut-2 \\
\hline \multirow[t]{2}{*}{ IV } & a. Secondary branch number distinctly high $\left(10\right.$ plant $\left.^{-1}\right)$ & $\mathrm{D}_{1} / 24-29$ \\
\hline & b. Secondary branch absent & 18 genotypes \\
\hline \multirow[t]{2}{*}{$\mathrm{V}$} & a. Leaf colour light green & Dhaka-1 \\
\hline & b. Leaf colour dark green & Mut-2,Mut-3 and Mut-5 \\
\hline \multirow[t]{2}{*}{ VI } & a. Stem pigmentation present & Zhingabadam \\
\hline & b. Stem pigmentation absent & 29 genotypes \\
\hline \multirow[t]{3}{*}{ VII } & a. Leaflet shape oblong-elliptic & $\mathrm{D}_{1} / 86-54$, Dhaka- $1, \mathrm{D}_{1} / 23-73$ \\
\hline & b. Leaflet shape lanceolate & M6/54-20 \\
\hline & c. Narrow-elliptic & 26 genotypes \\
\hline \multicolumn{3}{|c|}{ Flower characters } \\
\hline \multirow[t]{2}{*}{ VIII } & a. Two buds raceme ${ }^{-1}$ & $M_{6} / 36-24, M_{6} / 61-6$ \\
\hline & b. one bud raceme ${ }^{-1}$ & 28 genotypes \\
\hline \multirow[t]{2}{*}{ IX } & a. Calyx tube distinctly long $(5.04 \mathrm{~cm})$ & Mut-5 \\
\hline & b. Calyx tube short $(\leq 2.61 \mathrm{~cm})$ & $\mathrm{M}_{6} / 90-39, \mathrm{D}_{1} / 86-54$, Zhingabadam \\
\hline \multirow[t]{2}{*}{$X$} & a. Standard petal length long $(\geq 1.50 \mathrm{~cm})$ & $\mathrm{D}_{1} / 24-29$, Mut-2, Mut-5 \\
\hline & b. Standard petal length intermediate & 28 genotypes \\
\hline \multirow[t]{2}{*}{ XI } & a. Standard petal width high $(\geq 1.85 \mathrm{~cm})$ & Mut-2, Mut-5 \\
\hline & b. Standard petal width distinctly short $(\leq 1.22 \mathrm{~cm})$ & $\mathrm{D}_{1} / 24-29, \mathrm{D}_{1} / 11-60$ \\
\hline \multicolumn{3}{|c|}{ Pod characters } \\
\hline & a. Pod length long $(\geq 2.84 \mathrm{~cm})$ & Mut-2, Zhingabadam \\
\hline & b. Pod length short $(\leq 1.10 \mathrm{~cm})$ & 7 genotypes \\
\hline \multirow[t]{2}{*}{ XIII } & a. Pod three seeded & Zhingabadam \\
\hline & b. Pod two seeded & 29 genotypes \\
\hline \multirow[t]{2}{*}{ XIV } & a. Pod highly constricted & Mut-2, Mut-3 \\
\hline & b. Pod less constricted & 28 genotypes \\
\hline \multirow[t]{3}{*}{ XV } & a. Veins prominent & Zhingabadam \\
\hline & b. Veins intermediate & 6 genotypes \\
\hline & c. Veins small & 23 genotypes \\
\hline \multirow[t]{2}{*}{ XVI } & a. Pod beak present & Zhingabadam \\
\hline & b. Pod beak absent & 29 genotypes \\
\hline \multirow[t]{2}{*}{ XVII } & a. 100-pod weight distinctly high ( $\geq 187 \mathrm{~g})$ & Mut-2, Mut-3 \\
\hline & b. 100 -pod weight low $(\leq 80 \mathrm{~g})$ & 8 genotypes \\
\hline \multirow[t]{2}{*}{ XVIII } & a. 100-kernel weight high $(\geq 80 \mathrm{~g})$ & Mut-2, Mut-5 \\
\hline & c. 100 -kernel weight distinctly low $(\leq 28.67 \mathrm{~g})$ & $\mathrm{D}_{1} / 24-29$ \\
\hline
\end{tabular}




\section{Conclusion}

Only single genotype of the whole lot showed distinctively the longest primary branch and highest secondary branch number and small seed size ( $\left.\mathrm{D}_{1} / 24-29\right)$, highest primary branch number $\left(\mathrm{M}_{6} / 7-25\right)$, lowest primary branch number (Mut-2), highest leaflet length and light green leaf colour (Dhaka-1), presence of stem pigmentation and pod beak and highest number of seeds pod ${ }^{-1}$ (Zhingabadam), leaflet shape lanceolate (M6/54-20). In contrast, only two mutants of the lot showed distinctive shortest plant height ( $\mathrm{M}_{6} / 64-82$ and $\left.\mathrm{M}_{6} / 57-15\right)$, two buds raceme $^{-1}\left(\mathrm{M}_{6} / 36-24\right.$ and $\left.\mathrm{M}_{6} / 61-6\right)$, highest petal length (Mut-2 and Mut-5), highest pod length (Mut-2 and Zhingabadam), bolder pod and seed size and highly constricted pod (Mut-2 and Mut-3). All these mutants with the above distinguished characteristic featured for being ideal genetic markers and could be used in future breeding applications.

\section{References}

[1] Ashri A, Levy A. Spontaneous and induced Plasmon mutants in higher plant, Arachis hypogaea L. Genetica Agraria Monog. 1979;4: 71-76.

[2] Bayor TB, Dzomeku IK, Avornyo VK, Opoku-Agyeman MO. Morphological variation in Kersting's groundnut landraces from northern Ghana. Agric. Biol. J. North America. 2010; 1: 290-295. https://doi.org/10.5251/abjna.2010.1.3.290.295.

[3] Chandran K, Padya SM. Morphological characterization of Arachis species of section Arachis. Plant Genet. Resour. Newsl. 2000; 121: 38-41.

[4] Doku EV, Asiama O. A classification of Bambara groundnut cultivars based on variation in morphological characteristics. In: Proceedings of the First National Symposium on crop improvement in Ghana. Held at the Univ. Ghana, Legon, Accra on 22-24 August 1978. Doku E. V. (Ed.), CSIR. pp. 1978;19-27.

[5] Food and Agriculture Organization of the United Nations (FAO), FAOSTAT database. http://www.FAO.ORG.2016

[6] Gibbons RW, Bunting AH, Smart J. The classification of varieties of groundnut. Euphytica. 1972; 21: 78-85. https://doi.org/10.1007/BF00040550.

[7] Lara-Fioreze ACC, Tomaz CA, Fioreze SL, Pilon C, Zanotto MD. Genetic diversity among progenies of Crambe abyssinica Hochst for seed traits. Ind. Crops Prod. 2013; 42: 771-775. https://doi.org/10.1016/j.indcrop.2013.07.039.

[8] Latif MA, Rafii YM, Rahman MM, Talukdar MB. Microsatellite and minisatellite markers-based DNA fingerprinting and genetic diversity of blast and ufra resistant rice genotypes. Comptes Rendus Biol. 2011;334: 282-289. https://doi.org/10.1016/..crvi.2011.02.003.

[9] Latif MA, Rahman MM, Ali ME, Ashkani S, Rafii MY. Inheritance studies of SSR and ISSR molecular markers and phylogenetic relationship of rice genotypes resistant to tungro virus. Comptes Rendus Biol. 2013;336: 125-133. https://doi.org/10.1016/j.crvi.2012.12.002.

[10] Mondal MMA, Puteh AB. Effect of source of nitrogen on nitrate reductase activity and biomass productivity in peanut mutant. Res. Crops. 2013; 14: 507-510.

[11] Mouli C, Patil SH. Gamma-ray induced mutant with suppressed branches in peanut. J. Hered. 1976;67: 322-324. https://doi.org/10.1093/oxfordjournals.jhered.a108743.

[12] Ntundu WH, Shillah SA, Marandu WYF, Christiansen JL. Morphological diversity of Bambara groundnut [Vigna subterranean (L.) Verdc.] landraces in Tanzania. Genet. Resour. Crop Evol. 2006; 53: 367-378. https://doi.org/10.1007/s10722-004-0580-2.

[13] Pasquet RS, Schwedes S, Gepts P. Isozyme diversity in Bambara groundnut. Crop Sci. 1999; 39: 1228-1236. https://doi.org/10.2135/cropsci1999.0011183X003900040045x.

[14] Patil SH, Mouli C. X-ray induced asynaptic mutant in groundnut. Indian J. Exp. Biol. 1977; 15: 521-523.

[15] Patil SH. Induced mutations for improving quantitative characters of groundnut. Indian J. Genet. Plant Breed. 1972; 32: 451-459.

[16] Rafii MY, Shabanimofrad M, Puteri Edaroyati MW, Latif MA. Analysis of the genetic diversity of physic nut, Jatropha curcas L accessions using RAPD markers. Mol. Biol. Report. 2012; 39: 6505-6511. https://doi.org/10.1007/s11033-012-1478-2.

[17] Rohlf F. NTSYS-pc: Numerical Taxonomy System, version 2 1. Exeter Publishing Ltd., Setauket, NY, USA. 2002

[18] Russell DF. MSTAT-C Pakage Programme. Crop and Soil Science Department, Michigan University, USA. 1986.

[19] Upadhyaya HD. Phenotypic diversity in groundnut core collection assessed by morphological and agronomical evaluations. Genet. Resour. Crop Evol. 2003;50: 539- 550.

[20] Zivkovic B, Radovic J, Sokolovic D, Siler B, Banjanac T, Strbanovic R. Assessment of genetic diversity among alfalfa genotypes by morphometry, seed storage proteins and RAPD analysis. Ind. Crops Prod. 2012; 40: 285-291. https://doi.org/10.1016/j.indcrop.2012.03.027. 